\title{
Mitochondrial gene $C O X 2$ methylation and downregulation is a biomarker of aging in heart mesenchymal stem cells
}

\author{
XIGUANG SUN $^{1 *}$, ZILONG WANG $^{1 *}$, XIAOQIANG $\mathrm{CONG}^{2}$, YUDAN $\mathrm{LV}^{3}$, \\ ZHUO $\mathrm{LI}^{4}, \mathrm{LI} \mathrm{RONG}^{5}$, TIANYE YANG ${ }^{5}$ and DEHAI YU ${ }^{6}$

\begin{abstract}
Departments of ${ }^{1}$ Hand Surgery, ${ }^{2}$ Cardiology, ${ }^{3}$ Neurology and Neuroscience Center, ${ }^{4}$ Endocrinology and Metabolism, and ${ }^{5}$ Plastic and Reconstructive Surgery, The First Hospital of Jilin University, Changchun, Jilin 130021;

${ }^{6}$ Laboratory of Cancer Precision Medicine, The First Hospital of Jilin University, Changchun, Jilin 130061, P.R. China
\end{abstract}

Received February 16, 2020; Accepted October 19, 2020

DOI: $10.3892 / \mathrm{ijmm} .2020 .4799$

\begin{abstract}
The mitochondria have been proven to be involved in processes of aging; however, the mechansims through which mitoepigenetics affect the cytological behaviors of cardiomyocytes during the aging process are not yet fully understood. In the present study, two senescence models were constructed, replicative senescence (RS) and stress-induced premature senescence (SIPS), using human heart mesenchymal stem cells (HMSCs). First, the differences in age-related gene expression levels and telomere length were compared between the HMSCs in the RS and SIPS models by PCR. Subsequently, protein expression and the mitochondrial DNA (mtDNA) methylation status of cytochrome c oxidase subunit II (COX2) was measured by western blot analysis and bisulfite genomic sequencing (BSP). Finally, the value of the DNA methyltransferase (Dnmt) inhibitor, 5-aza-2'-deoxycytidine (AdC), in delaying the senescence of HMSCs was evaluated. It was found that the p16, p27 and p53 mRNA expression levels increased in the senescent cells, whereas p 21 mRNA expression did not. It was also found that telomere shortening only occurred in the RS model, but not in the SIPS model. Along with the senescence of HMSCs, COX2 gene methylation increased and its protein expression level significantly decreased. It was demonstrated that AdC inhibited COX2 methylation and downregulated COX 2 expression. The addition of exogenous COX2 or the administration of AdC promoted cell proliferation and delayed cell aging. On the whole, the present study demonstrates that COX2 methylation and downregulation are biomarkers of HMSC senescence. Thus, COX2 may have
\end{abstract}

Correspondence to: Professor Dehai Yu, Laboratory of Cancer Precision Medicine, The First Hospital of Jilin University, 519 Dongminzhu Street, Changchun, Jilin 130061, P.R. China

E-mail: yudehai@jlu.edu.cn

*Contributed equally

Key words: mitochondria, cytochrome c oxidase subunit II, methylation, senescence, mesenchymal stem cell potential for use as a therapeutic target of cardiovascular diseases and this warrants further investigation.

\section{Introduction}

Aging is a universal biological phenomenon known to induce a declines in the physiological capacity of a number of tissues, including the cardiovascular system. As the elderly population is increasing worldwide, age has become the dominant risk factor for the development of cardiovascular diseases (1). To prevent age-related cardiac diseases, further insight should be gained from the investigation of the processes involved in cardiac aging.

The mitochondria constitute the central metabolic hub in cells and are the gateway to aerobic metabolism (2). The mitochondria have also been proven to be intimately involved in signal transduction and aging processes, particularly in the aging of the heart $(3,4)$. The mitochondria also undergo several alternations in aged cardiomyocytes, such as reductions in the number and area of the inner membrane, an increase in the volume fraction and alternations in shape (5-9).

Cytochrome c oxidase (COX), also known as complex IV, is the terminal enzyme of the electron transport chain, catalyzing the reduction of oxygen to water. COX is composed of at least 15 subunits, 12 of which are encoded by nuclear DNA and 3 of which are encoded by mitochondrial DNA (mtDNA) (10). The mtDNA-encoded subunits, COX1 (MT-COI), COX2 (MT-COII) and COX3 (MT-COIII), form the catalytic core of the enzyme $(11,12)$.

Similar to the methylation of genomic DNA, mtDNA has been found to be CpG-methylated, which plays an important role in mitochondrial gene regulation $(13,14)$. A previous study by the authors reported a phenomenon of decreased methylation at several methylation hotspots on the mtDNA of senescent human heart mesenchymal stem cells (HMSCs) (15). In the present study, using bisulfite genomic sequencing (BSP), the methylation of the COX2 gene was detected in aged HMSCs. In addition, the effects of COX2 on cell senescence and proliferation were investigated. The present study may provide a deeper understanding of the mechanisms through which the mitochondria function during the aging process. 


\section{Materials and methods}

Cell culture. HMSCs were isolated and were a kind gift from Dr Ji-Fan Hu of Stanford University (15). The study group of Dr Ji-Fan Hu obtained the ethical approval and informed consent for the isolation of the HMSCs. The characteristics of the HMSCs were also measured and described in this previous study (15). HMSCs (G0) were cultured in normal DMEM (Biological Industries, Inc.) containing 10\% fetal bovine serum (FBS, Biological Industries, Inc.) and antibiotics (Biological Industries, Inc.) and incubated at $37^{\circ} \mathrm{C}$ in a $5 \% \mathrm{CO}_{2}$ humidified atmosphere.

5-Aza-2'-deoxycytidine (AdC, MCE Corporation) was used as a DNA methylation inhibitor. AdC solution was prepared with DMSO (Sigma-Aldrich; Merck KGaA) and the final working concentration in the culture medium was $1 \mu \mathrm{M}$, as described in a previous study (16).

Establishment of replicative senescence $(R S)$ and stress-induced premature senescence (SIPS) models. For the RS model, HMSCs at the G0 phase were continuously cultured in normal DMEM in $10 \mathrm{~cm}$ dishes. Cells were detached and passaged at the ratio of 1:2 when the confluence reached $70 \%$. The generation of HMSCs $(\mathrm{Gn})$ was defined using the cell passage number. A strategy was adopted by chronically exposing HMSCs to low levels of oxidative stress (50 $\mu \mathrm{M} \mathrm{H}_{2} \mathrm{O}_{2}$; Sigma-Aldrich; Merck KGaA) and a low serum environment (5\% FBS) to establish the SIPS model, which was also described in a previous study by the authors (15).

Staining of senescence-associated $\beta$-galactosidase $(S A-\beta-G a l)$. Cellular senescence was visualized and quantified by measuring the activity of $\beta$-Gal. HMSCs in a 6-well plate were fixed with $3 \%$ formaldehyde (Beijing Chemical Works) for $3 \mathrm{~min}$ at room temperature. After washing with PBS for $2 \times 5$ min, cell senescence was determined with the Senescence $\beta$-Galactosidase Staining kit according to the manufacturer's protocol (Beyotime Institute of Biotechnology, Inc.). Following overnight incubation at $37^{\circ} \mathrm{C}$, HMSCs with the SA- $\beta$-Gal staining were assessed using a microscope-mounted camera (IXplore, Olympus Corporation).

Detection of telomere length in senescent HMSCs. The relative length of telomeres in the HMSCs was measured by real-time fluorescence quantitative polymerase chain reaction (qPCR) as previously described $(17,18)$, with certain modifications. Briefly, total cellular DNA was extracted using the Qiagen DNeasy Blood \& Tissue kit (Qiagen, Inc.). DNA was diluted to $35 \mathrm{ng} / \mu 1$ and was heated at $95^{\circ} \mathrm{C}$ for $5 \mathrm{~min}$ and chilled on ice for $5 \mathrm{~min}$. The DNA samples were then placed in a $20 \mu \mathrm{l} \mathrm{qPCR}$ reaction system containing $10 \mu \mathrm{l} 2 \mathrm{X}$ SYBR premixed buffer (Genstar Technologies Company Inc.), and $2 \mu \mathrm{l}$ forward and reverse primers. The sequences of the primers were as follows: Telomere forward, 5'-GGTTTTTGA GGGTGAGGGTGAGGGTGAGGGTGAGGGT-3' (100 nM) and reverse, 5'-TCCCGACTATCCCTATCCCTTCCCTAT CCCTATCCCTA-3' (300 nM); $\beta$-globin forward, 5'-GCTTCT GACACAACTGTGTTCACTAGC-3' $(150 \mathrm{nM})$ and reverse, 5'-CACCAACTTCATCCACGTTCACC-3' (150 nM). The PCR amplification process was as follows: One cycle at $95^{\circ} \mathrm{C}$ for $10 \mathrm{~min}, 40$ cycles at $95^{\circ} \mathrm{C}$ for $15 \mathrm{sec}, 56^{\circ} \mathrm{C}$ for $30 \mathrm{sec}$, and $72^{\circ} \mathrm{C}$ for $30 \mathrm{sec}$ (ABI SepOnePlus). Telomere length was estimated by calculating the relative ratio between the copies of telomere and the copies of $\beta$-globin. The qPCR results were calculated using $2^{-\Delta \Delta \mathrm{Cq}}$ method (19).

Relative qualification of mtDNA copy number. Total cellular DNA was extracted as described above. The change in mtDNA copy number was evaluated by qPCR targeting the $\beta$-globin gene and the mitochondrial ND1 gene as previously described by Ding et al (20). Primers and probes were as follows: For the $\beta$-globingene: Forward,5'-CTGGGCATGTGGAGACAGAGA AGACT-3' and reverse, 5'-AGGCCATCACTAAAGGCACCG AGC-3'; probe: 5'(FAM)-CCCTTAGGCTGCTGGTGGTCT ACCCTT-(TAMRA)3'. For mitochondrial ND1 gene: Forward, 5'-GACGCCATA AAACTCTTCACCAA-3' and reverse, 5'-AGGTTGAGGTTGACCAGGGG-3'; probe: 5' (FAM)-CCA TCACCC TCT ACA TCACCG CCC -(TAMRA) 3'. The reaction was conducted in a $20 \mu 1$ system containing $2 \mu 1$ DNA ( 70 ng), $10 \mu$ l Faststart TaqMan Probe Master (ROX) (Roche Diagnostics, Inc.), $300 \mathrm{nM}$ forward and reverse primers and $100 \mathrm{nM}$ TaqMan probe. The PCR amplification process was as follows: One cycle at $95^{\circ} \mathrm{C}$ for $10 \mathrm{~min}, 40$ cycles at $95^{\circ} \mathrm{C}$ for $15 \mathrm{sec}$ and $60{ }^{\circ} \mathrm{C}$ for $1 \mathrm{~min}$. The qPCR results were calculated using the $2^{-\Delta \Delta \mathrm{Cq}}$ method.

Reverse transcription PCR (RT-PCR). The age-related gene expression levels of $p 16, p 27, p 53$ and $p 21$ were determined by RT-PCR. Total RNA was extracted from the HMSCs using the EasyPure RNA Purification kit (TransGen Biotech). In total, 1,000 ng RNA was reverse transcribed with TransScript All-in-One First-Strand cDNA Synthesis SuperMix for qPCR (One-Step gDNA Removal) (TransGen Biotech). The primers were as follows: $p 16$ forward, 5'-ACCAGAGGCAGTAACCAT GC-3' and reverse, 5'-GACCTTCGGTGACTGATGATC-3'; $p 27$ forward, 5'-GCCTCAGAAGACGTCAAACGT-3' and reverse, 5'-TGTCCATTCCATGAAGTCAGC-3'; $p 53$ forward, 5'-CTC AGCATCTTATCCGAGTGG-3' and reverse, 5'-GCAGGAACT GTTACACATGTAG-3'; $p 21$ forward, 5'-GTGGACCTGTCA CTGTCTTGTAC-3' and reverse, 5'-GCTTCCTCTTGGAGA AGATCAGC-3'; $\beta$-actin forward, 5'-CAGGTCATCACCATT GGCAATGAGC-3' and reverse, 5'-CGGATGTCCACGTCA CACTTCATGA-3'. RT-PCR was conducted on an Eppendorf Mastercycler (Eppendorf Shanghai International Trade Co. Ltd.) and was based on the instructions provide with RealStar Green Fast Mixture (GenStar). The reaction mixture contained the following: $2 \mu \mathrm{l}$ cDNA, $4 \mu 1$ 2X Taq PCR StarMix buffer and $2 \mu \mathrm{l}$ of each primer. The thermocycling conditions were as follows: Initial denaturation at $98^{\circ} \mathrm{C}$ for $3 \mathrm{~min}$, followed by 25 cycles (for $\beta$-actin) or 31 cycles (for other genes) of $95^{\circ} \mathrm{C}$ for $15 \mathrm{sec}, 62^{\circ} \mathrm{C}$ for $15 \mathrm{sec}$ and $72^{\circ} \mathrm{C}$ for $15 \mathrm{sec}$, and a final extension step at $72^{\circ} \mathrm{C}$ for $5 \mathrm{~min}$. The reaction products were separated on a 3\% agarose gel and visualized by GelStain (TransGen). Densitometric analysis was performed using Quantity One software (version 4.6; Bio-Rad Laboratories, Inc.).

Gene synthesis, plasmid construction and transfection. The COX2 gene was optimized according to nuclear format and commercially synthesized (Sangon Biotech (Shanghai) Co., Ltd.). The synthesized gene was sub cloned into the Sal1/Xho1 
A

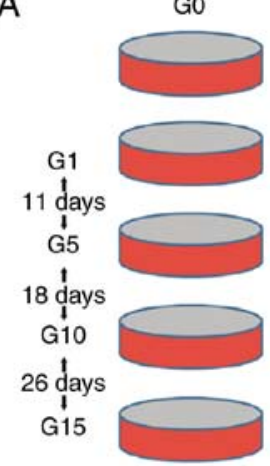

D

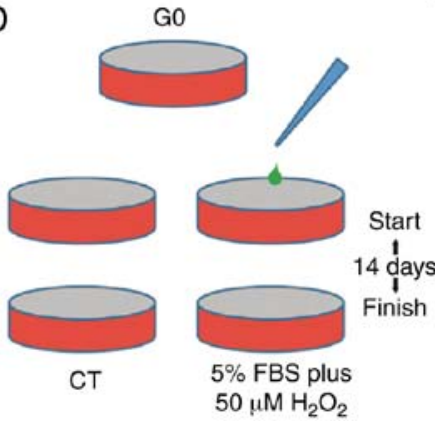

B

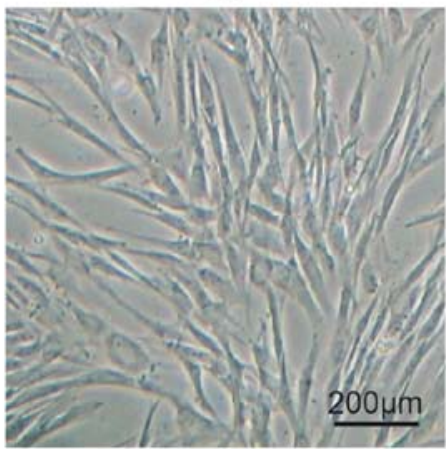

E

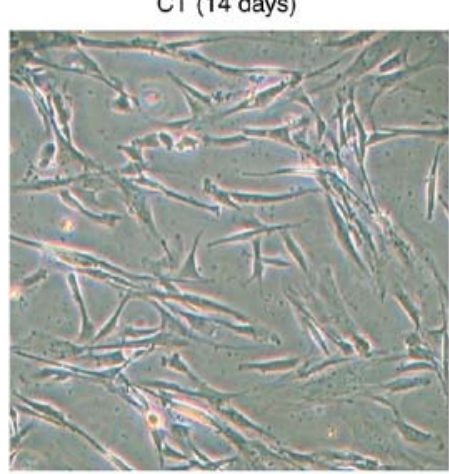

C

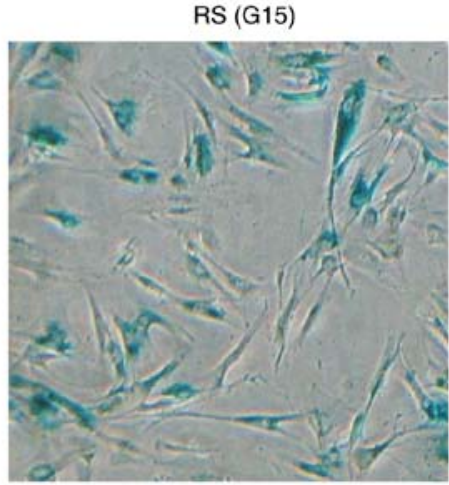

$\mathrm{F}$

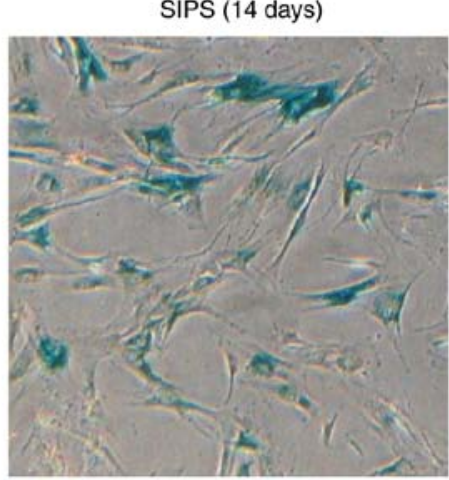

Figure 1. Construction of RS and SIPS models of HMSCs. (A) Diagram illustrating the inducing process of the RS model. HMSCs were continually cultured in normal medium from G0 to G15. (B and C) SA- $\beta$-Gal staining showing the G15 HMSCs displaying obvious senescence following culture for 55 days. (D) Diagram illustrating the induction process of the SIPS model by $5 \%$ FBS plus $50 \mu \mathrm{M} \mathrm{H}_{2} \mathrm{O}_{2}$. (E and F) SA- $\beta$-Gal staining showing the premature senescence of HMSCs. HMSCs, heart mesenchymal stem cells; RS, replicative senescence; SIPS, stress-induced premature senescence.

restriction site of the mitochondria targeting plasmid vector, pCMV-myc-mito (Invitrogen; Thermo Fisher Scientific, Inc.), to form a new pCMV-COX2-myc-mito plasmid. HMSCs were transfected with $2 \mu \mathrm{g}$ plasmid in a 6 -well plate using Lipofectamine 3000 reagent (Invitrogen; Thermo Fisher Scientific, Inc.) according to the manufacturer's protocol. Empty vector was also transfected into HMSCs as a negative control. Stable transfectants were selected using neomycin (Invitrogen; Thermo Fisher Scientific, Inc.) within 2 weeks, and after that, subsequent experiments were carried out.

Protein extraction and western blot analysis. Whole cell protein was extracted using the Protein Extraction kit (Beyotime Institute of Biotechnology, Inc.). Equal amounts of $25 \mu \mathrm{g}$ protein from each sample were separated by $5-12 \%$ SDS polyacrylamide gel electrophoresis and were transferred to $0.45 \mu \mathrm{m}$ PVDF membranes (EMD Millipore). Membranes were incubated in $5 \% \mathrm{BSA} / \mathrm{TBST}$ solution at $37^{\circ} \mathrm{C}$ for $1 \mathrm{~h}$ and washed in TBST for 3x5 min. Membranes were then incubated with anti-COX2 (1:1,000 ab110258, Abcam), anti-myc-Tag (1:1,000, 2267, Cell signaling Technology, Inc.) and anti- $\beta$-actin (1:3,000, ab8226, Abcam) antibodies at $4{ }^{\circ} \mathrm{C}$ overnight. Following incubation with the primary antibodies, the membranes were incubated with HRP-coupled goat anti-mouse secondary antibody (1:3,000, A0216, Beyotime Institute of Biotechnology, Inc.) at room temperature for $1 \mathrm{~h}$. Immunoreactivity was detected using ECL luminescence reagent [Sangon Biotech (Shanghai) Co., Ltd.]. Densitometric analysis was performed using Quantity One software (version 4.6.2, Bio-Rad Laboratories, Inc.).
MtDNA methylation analysis. HMSC mitochondria were isolated using the Tissue Mitochondria Isolation kit (Beyotime Institute of Biotechnology, Inc.) and mtDNA was extracted. mtDNA methylation was detected by BSP. According to the manufacturer's protocol, 1,000 ng mtDNA in total was used for bisulfite conversion with the EpiTect Bisulfite kit (Qiagen, Inc.). The normal and converted COX2 mtDNA was amplified by PCR reaction using the following primers: Forward primer, 5'-ATTTTGTTAAAGTTAAATTATAGG-3' and reverse primer, 5'-TTAATTCTCTTAATCTTTAACTTA-3'. The thermocycling conditions were as follows: Initial denaturation at $98^{\circ} \mathrm{C}$ for $5 \mathrm{~min}$, followed by 30 cycles of $95^{\circ} \mathrm{C}$ for $20 \mathrm{sec}, 62^{\circ} \mathrm{C}$ for $20 \mathrm{sec}$ and $72^{\circ} \mathrm{C}$ for $100 \mathrm{sec}$, and a final extension step at $72^{\circ} \mathrm{C}$ for $10 \mathrm{~min}$. The reaction products were separated on a $1 \%$ agarose gel and recycled using AxyPrep DNA Gel Extraction kit (Hangzhou Axygen Biotechnology).

The recycled DNA molecules were linked with the pJET1.2/blunt vector supplied with the CloneJET PCR Cloning kit (K1231, Thermo Fisher Scientific, Inc.) and sequenced using Sanger method [Sangon Biotech (Shanghai) Co., Ltd.]. The methylation status of the 29th CpG site on the COX2 gene was analyzed. The percentage of methylated $\mathrm{CpG}=$ the number of unconverted $\mathrm{C}$ in $\mathrm{CpG} /$ the total number of $\mathrm{CpG} \times 100 \%$.

Cell proliferation assay. Cell proliferation was determined by WST-1 assay (Beyotime Institute of Biotechnology, Inc.). Briefly, $5 \times 10^{3}$ HMSCs were planted into a well of 96-well plate and cultured at $37^{\circ} \mathrm{C}$ for $24 \mathrm{~h}$. According to the manufacturer's instructions, $20 \mu \mathrm{l}$ WST-1 were added to $200 \mu \mathrm{l}$ cell culture 

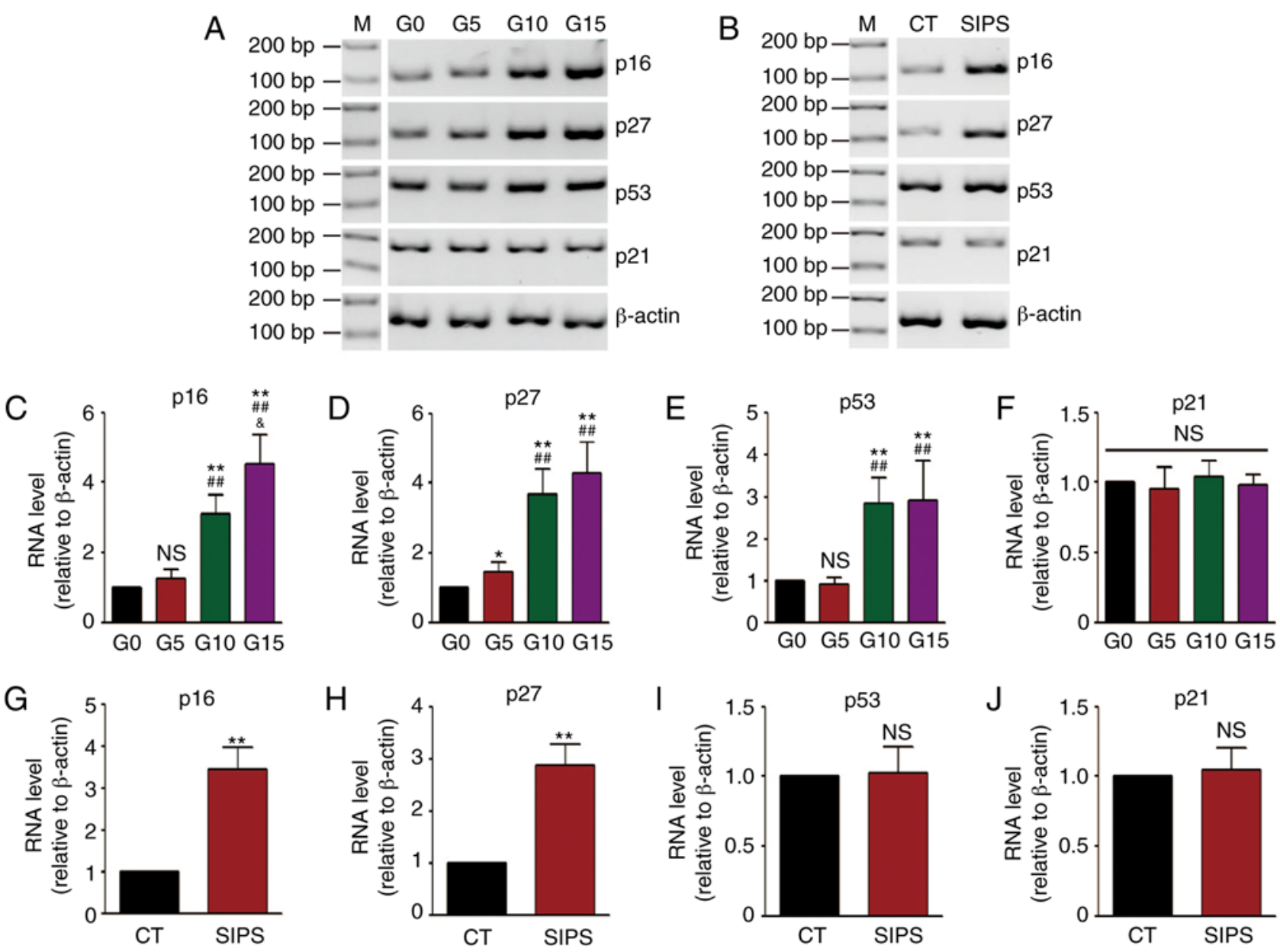

Figure 2. Detection of age-related genes expression by RT-PCR. (A and B) RT-PCR showing $p 16, p 27, p 53$ and $p 21 \mathrm{mRNA}$ expression in the (A) RS and (B) SIPS models. (C-F) Densitometric analysis showing that the $p 16, p 27$ and $p 53 \mathrm{mRNA}$ expression levels were increased in the RS model. "P<0.05 and ${ }^{* *} \mathrm{P}<0.01$ as compared with the G0 group; ${ }^{\# \#} \mathrm{P}<0.01$ as compared with the G5 group; ${ }^{\circledR} \mathrm{P}<0.05$ as compared with the G10 group. (G-J) Densitometric analysis showing that the $p 16$ and $p 27$ mRNA expression levels were increased in the SIPS model. ${ }^{* *} \mathrm{P}<0.01$ as compared with the CT group. M, molecular weight; NS, not significant; HMSCs, heart mesenchymal stem cells; RS, replicative senescence; SIPS, stress-induced premature senescence.

medium and incubated at $37^{\circ} \mathrm{C}$ in the dark for $2.5 \mathrm{~h} . \mathrm{OD}_{450}$ and $\mathrm{OD}_{630}$ were measured using a microplate reader (BioTek Instruments, Inc.).

Statistical analysis. SPSS 19.0 (SPSS Inc.) was used for the analysis of the data. All data were obtained from at least 3 independent experiments and expressed as the means \pm standard deviation (SD). Statistical significance between groups was determined using the Student's t-test or one-way ANOVA followed by the LSD or Tukey's post hoc test. A value of $\mathrm{P}<0.05$ was considered to indicate a statistically significant difference.

\section{Results}

Senescence of HMSCs in the RS and SIPS model. In the RS model, the HMSCs were continually cultured from G0 to G15, lasting for 55 days (Fig. 1A). In the SIPS model, the HMSCs were cultured in low FBS plus $50 \mu \mathrm{M} \mathrm{H}_{2} \mathrm{O}_{2}$ for 14 days (Fig. 1D). The HMSCs from the G0/G15 and CT/SIPS groups were stained for SA- $\beta$-Gal. As shown in Fig. $1 \mathrm{~B}, \mathrm{C}, \mathrm{E}$ and F, the HMSCs from the G15 and SIPS groups exhibited a typical senescence-like morphology and stained positive for $\beta$-Gal.
Subsequently, age-related gene expression levels were detected. The mRNA levels of 4 genes, including $p 16, p 27, p 53$ and $p 21$ were measured by RT-PCR. The results of RT-PCR of the HMSCs in the RS and SIPS models are shown in Fig. 2A and $\mathrm{B}$, and the results of densitometric analysis of RT-PCR are shown in Fig. 2C-J. It was found that the mRNA levels of the $p 16, p 27$ and $p 53$ genes were increased along with cell senescence in the RS model, while the p16 and $p 27$ levels were increased in the SIPS model.

Since telomere length is considered a biomarker of chronological aging (21), the present study also detected this the RS and SIPS models. The results of qPCR revealed that the telomere length of the HMSCs gradually became shorter in the RS model (Fig. 3A), but not in the SIPS model (Fig. 3B).

COX2 methylation and protein expression in senescent HMSCs. It has been reported that COX plays a critical role in age-associated cell dysfunction (10). In a previous study, the authors demonstrated that COX1 methylation decreased and COX1 mRNA expression was significantly upregulated following senescence (15). The present study wished to determine the mechanisms through COX2 mtDNA 

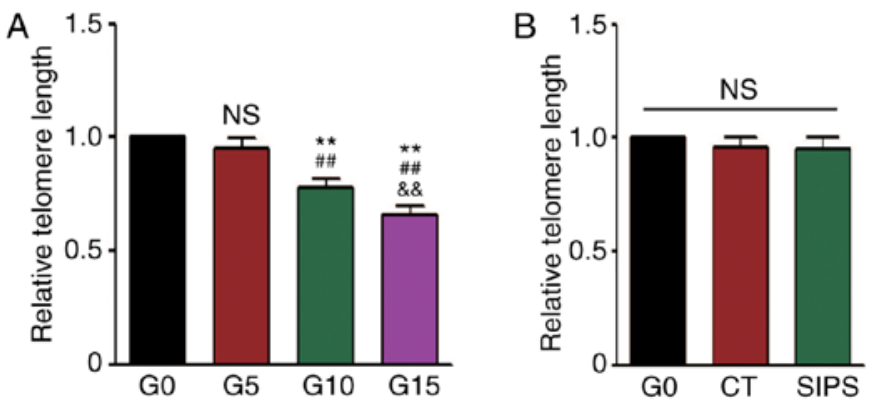

Figure 3. Detection of the relative telomere length of HMSCs. (A) qPCR result showing that the relative telomere length of HMSCs was significantly decreased in the RS model. ${ }^{* *} \mathrm{P}<0.01$ as compared with the G0 group; ${ }^{\# \# ~} \mathrm{P}<0.01$ as compared with the G5 group; \&\& $<<0.01$ as compared with the G10 group. (B) The relative telomere length of HMSCs was not altered in the SIPS model. NS, not significant; HMSCs, heart mesenchymal stem cells; RS, replicative senescence; SIPS, stress-induced premature senescence.

A

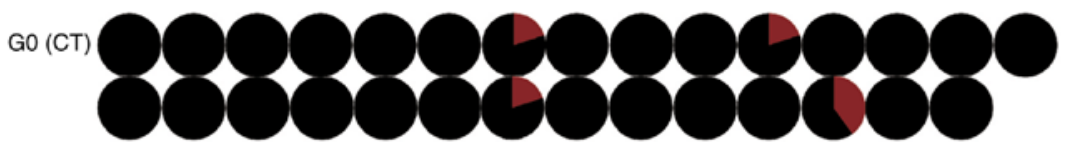

CpG1-15

CpG16-29

$\mathrm{B}_{\mathrm{G} 10}$

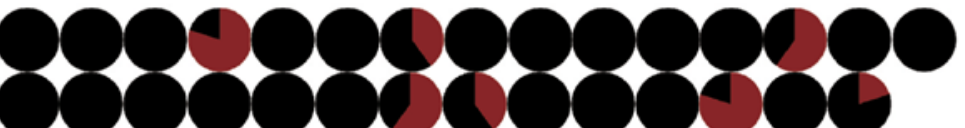

CpG1-15

CpG16-29

$\mathrm{C}_{\mathrm{G} 15}$

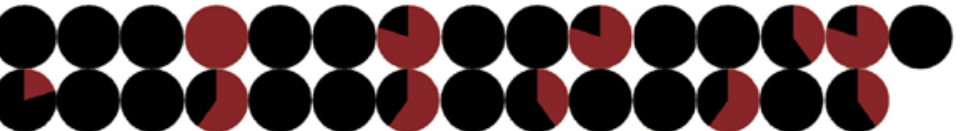

CpG1-15

CpG16-29

$D_{\text {SIPS }}$

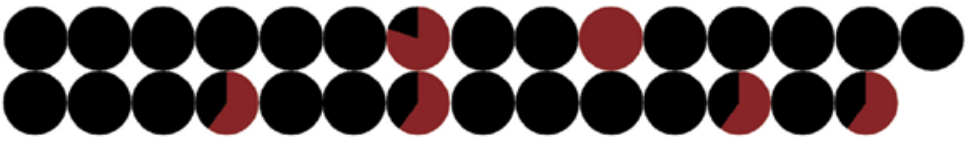

CpG1-15

CpG16-29
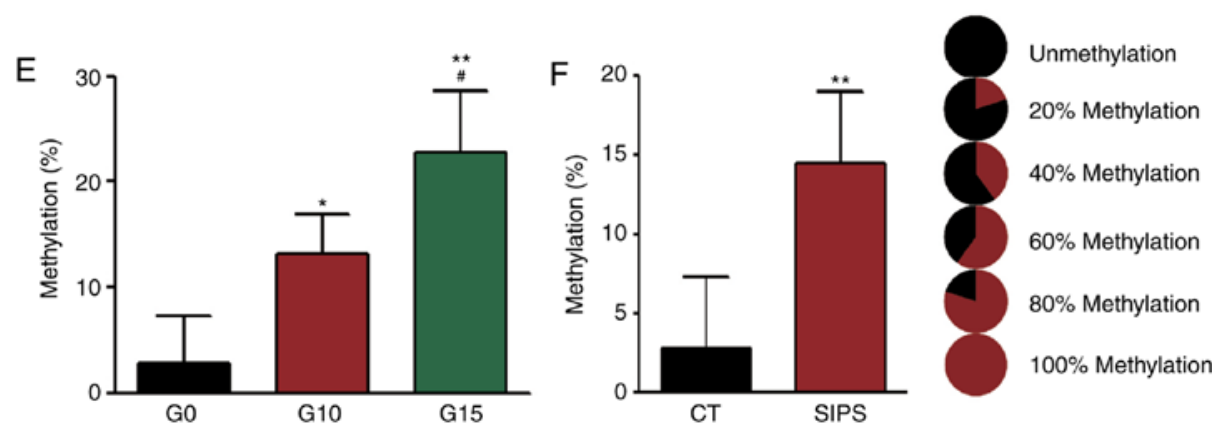

Figure 4. Detection of $C O X 2$ methylation using BSP. (A-D) Pie charts illustrating the methylation ratio of $29 \mathrm{CpG}$ sites on the COX2 gene. (E) Comparison of the methylation ratio among G0, G10 and G15 HMSCs. COX2 methylation was increased along with HMSC senescence. (F) COX2 methylation was also increased in the SIPS model. " $\mathrm{P}<0.05$ and ${ }^{*} \mathrm{P}<0.01$ as compared with the G0 or CT group; ${ }^{~} \mathrm{P}<0.01$ as compared with the G10 group. HMSCs, heart mesenchymal stem cells; RS, replicative senescence; SIPS, stress-induced premature senescence; BSP, bisulfite genomic sequencing.

methylation is altered in senescent HMSC. It was found that in the RS model, the results of BSP revealed that the methylation rate of the G0 HMSCs was $2.76 \%$ (Fig. 4A), that of G10 HMSCs was $13.1 \%$ (Fig. 4B) and that of G15 HMSCs was $22.76 \%$ (Fig. 4C), while in the SIPS model, the methylation rate of the senescent HMSCs was $14.48 \%$ (Fig. 4D).

Subsequently, the expression status of the $C O X 2$ gene was analyzed. Considering that the mitochondria number may change in cells and thus affect protein quantity, the mtDNA copy number was first measured by qPCR. It was found that although the mtDNA copy numbers in G10, G15 and SIPS
HMSCs decreased slightly, this decrease was not significant (Fig. 5A and B). The present study then detected the protein levels of COX2 in the RS and SIPS models by western blot analysis (Fig. 5C and D). The expression levels of COX2 were then evaluated using the ratio between the gray value of blots and the mtDNA copy number. As shown in Fig. 5E and F, the results indicated that $\mathrm{COX} 2$ expression decreased along with HMSC senescence in both the RS and SIPS models.

Overexpression of $\mathrm{COX} 2$ delays HMSC senescence. Since the COX2 level decreased along with cell senescence, exogenous 


\section{A}
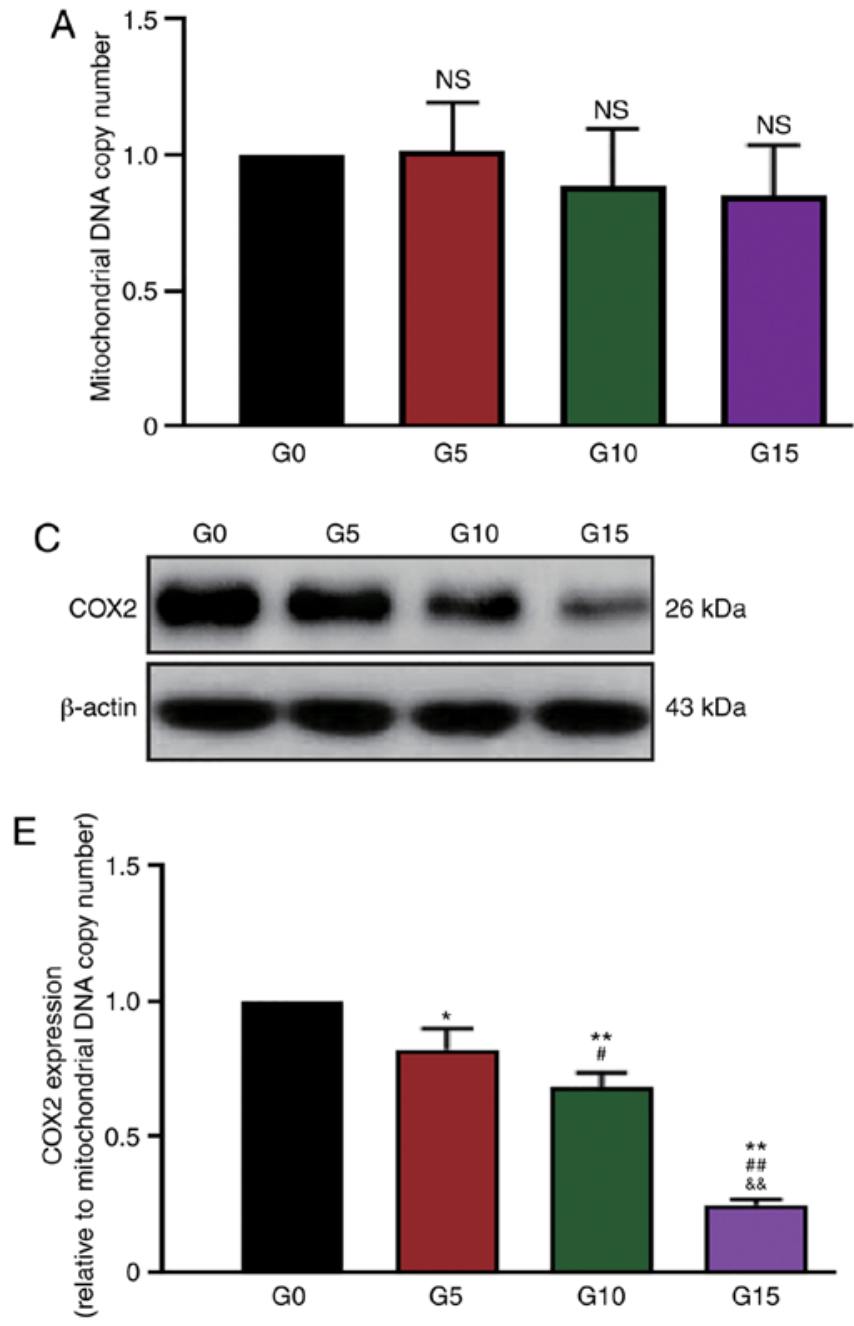

B

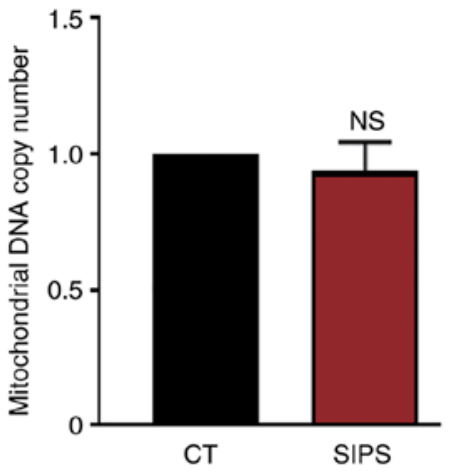

D

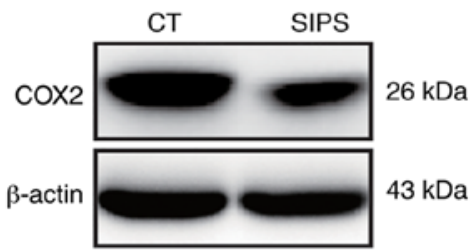

F

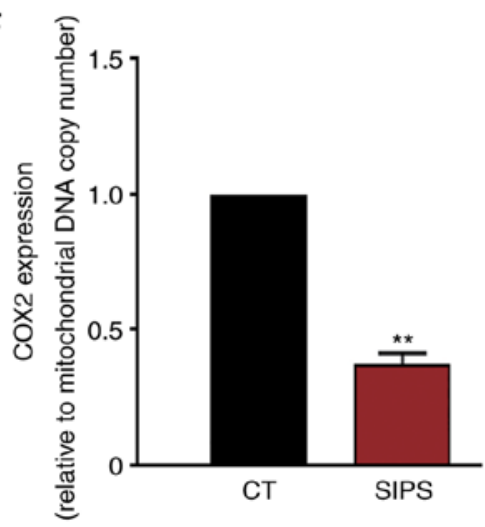

Figure 5. Analysis of the COX2 protein expression. mtDNA copy numbers of the COX2 were detected by qPCR and protein quantities were detected by western blot analysis. The expression levels of COX2 were evaluated using the ratio between the gray value of blots and the mtDNA copy number. (A and B) Relative mtDNA copy numbers in RS and SIPS cells were not altered significantly. (C and D) COX2 protein quantity in the RS and SIPS models; (E and F) statistical analysis results indicating that the COX2 protein expression level decreased along with HMSC senescence. " $\mathrm{P}<0.05$ and ${ }^{* *} \mathrm{P}<0.01$ as compared with the G0 or $\mathrm{CT}$ group; ${ }^{\#} \mathrm{P}<0.05$ and ${ }^{\# \#} \mathrm{P}<0.01$ as compared with the $\mathrm{G} 5$ group; \&\& $\mathrm{P}<0.01$ as compared with the G10 group. mtDNA, mitochondrial DNA; HMSCs, heart mesenchymal stem cells; RS, replicative senescence; SIPS, stress-induced premature senescence; COX2, cytochrome c oxidase subunit II.

COX2 protein was introduced into HMSC to determine whether it delays the aging process of HMSCs. The COX2 expression plasmid, pCMV-COX2-myc-mito (Fig. 6A and B), was transfected into the G1 HMSCs and stable transfectants were selected. The transfection efficiency was confirmed by western blot analysis using anti-myc antibodies (Fig. 6C). Stable HMSCs were also cultured in 5\% FBS and $50 \mu \mathrm{M} \mathrm{H}_{2} \mathrm{O}_{2}$ conditioned medium for 14 days. The results of WST-1 assay revealed that the $\mathrm{COX} 2$ transfectants grew at a faster rate than the normal HMSCs (Fig. 6D). SA- $\beta$-Gal staining revealed that the overexpression of COX2 significantly delayed the aging process of HMSCs (Fig. 6E-G).

AdC decreases COX2 methylation and attenuates HMSC senescence. To determine whether COX 2 mtDNA methylation is associated with cellular senescence, HMSCs were treated with AdC. As shown in Fig. 7A-C, COX2 mtDNA methylation in the G10, G15 and SIPS HMSCs was decreased $(\mathrm{P}<0.05$, pie charts are not shown). The COX2 protein expression levels were increased simultaneously $(\mathrm{P}<0.01$, Fig. $7 \mathrm{D}$ and $\mathrm{E}$; the
mtDNA copy number is not shown). It was also found that AdC treatment significantly increased the proliferation of the senescent HMSC (Fig. 7F-H). Importantly, SA- $\beta$-Gal staining revealed that $\mathrm{AdC}$ treatment also delayed the aging process of the HMSCs (Figs. 1C and F, and 7I-L).

\section{Discussion}

Due to increasing longevity and declining fertility, it has been predicted that almost one in four individuals will be 65 years or older by the year 2035 (22). There is a consensus that age is a dominant risk factor for the development of cardiac diseases, which is the leading cause of mortality worldwide (23). Therefore, there is an urgent need to elucidate the molecular mechanisms of age-related cardiac diseases and to develop novel intervention techniques.

In the present study, two types of aging models were constructed, RS and SIPS. RS is a state where normal somatic cells lose their replicative capacity in vitro and reach the Hayflick limit (24). SIPS is often used as a model to study 


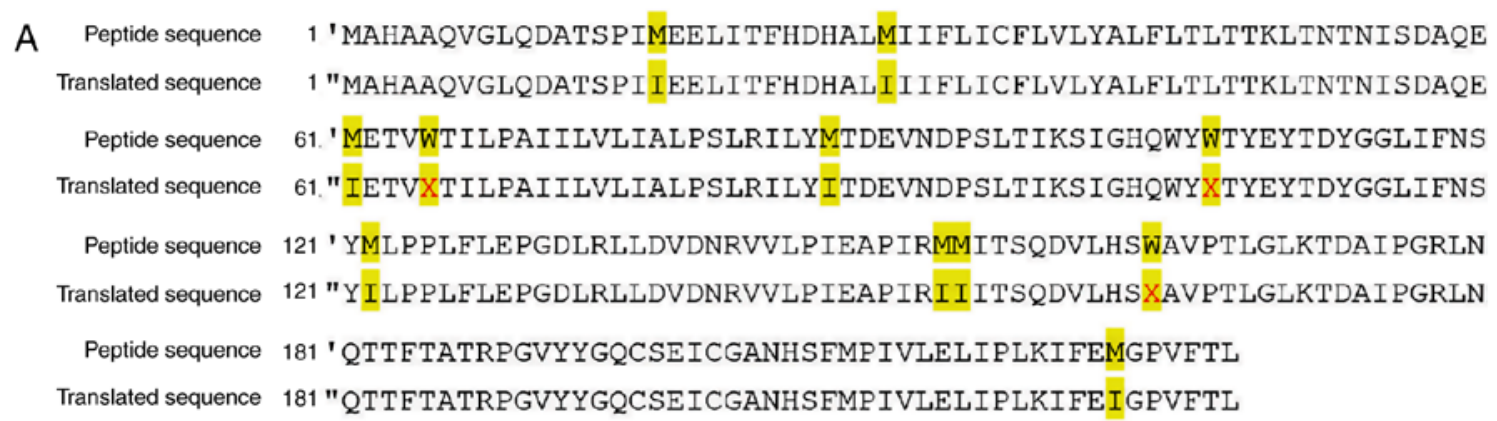

B 7561

AtgGC ACATGCAgCG CAAGTAGgtC TACAAGACGC 35

7621 TACTTCCCCT ATCATGGAAG AGCTTATCAC CTTTCATGAT CACGCCCTCA TGATCATTTT 95

7681 CCTTATCTGC TTCCTAGTCC TGTATGCCCT TTTCCTAACA CTCACAACAA AACTAACTAA 155

7741 TACTAACATC TCAGACGCTC AGGAAATGGA AACCGTCTGG ACTATCCTGC CCGCCATCAT 215

7801 CCTAGTCCTC ATCGCCCTCC CATCCCTACG CATCCTTTAC ATGACAGACG AgGTCAACGA 275

7861 TCCCTCCCTT ACCATCAAAT CAATTGGCCA CCAATGGTAC TGGACCTACG AGTACACCGA 335

7921 CTACGGCGGA CTAATCTTCA ACTCCTACAT GCTTCCCCCA TTATTCCTAG AACCAGGCGA 395

7981 CCTGCGACTC CTTGACGTTG ACAATCGAgt AgTACTCCCG ATTGAAGCCC CCATTCGTAT 455

8041 GATGATTACA TCACAAGACG TCTTGCACTC ATGGGCTGTC CCCACATTAG GCTTAAAAAC 515

8101 AGATGCAATT CCCGGACGTC TAAACCAAAC CACTTTCACC GCTACACGAC CGGGGGTATA 575

8161 CTACGgTCAA TGCTCTGAAA TCTGTGGAgC AAACCACAGT TTCATGCCCA TCGTCCTAGA 635

8221 ATTAATTCCC CTAAAAATCT TTGAAATGgG GCCCGTATTT ACCCTA
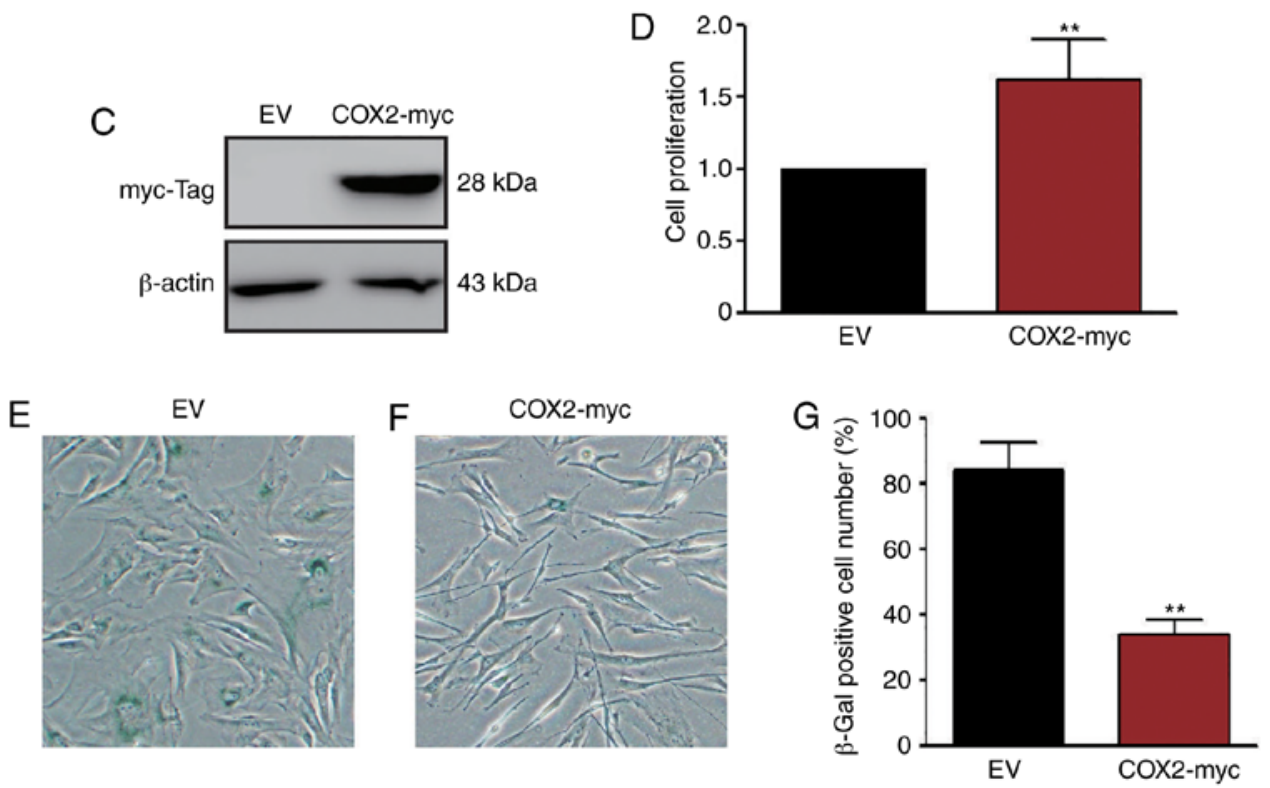

Figure 6. COX2 overexpression delays HMSC senescence. COX2 gene sequence was optimized and transformed according to the genetic code of nuclear DNA. The transformed DNA was subcloned into mitochondrial protein expressing vector and transfected into HMSCs. (A) Comparison of the COX2 amino acid sequence and the translated peptide sequence according to the genetic code of nuclear DNA. Those differential amino acids were highlighted by orange boxes. Stop code was symbolized by X. (B) mtDNA sequence was transformed accordingly to meet the variation of amino acids. Orange boxes: TGA (stop code) was replaced by TGG (tryptophan, W) and ATA (isoleucine, I) was replaced by ATG (methionine, M). (C) COX2 overexpression in HMSCs was confirmed by western blot analysis. (D) WST-1 assay indicated that COX2 overexpression promoted cell proliferation. (E-G) SA- $\beta$-Gal staining result showing that COX2 overexpression delayed the senescence of HMSCs. ${ }^{* *} \mathrm{P}<0.01$ as compared with the EV group. mtDNA, mitochondrial DNA; HMSCs, heart mesenchymal stem cells; COX2, cytochrome c oxidase subunit II.

aging as it shares the characteristics of senescent cells, such as increased levels of oxidative DNA damage, accelerated telomere shortening and changes in cell-cycle regulation (25).
However, there are still some differences between RS and SIPS, such as phenotypes of protein and gene expression (26). In the present study, 4 age-related gene expression profiles were 

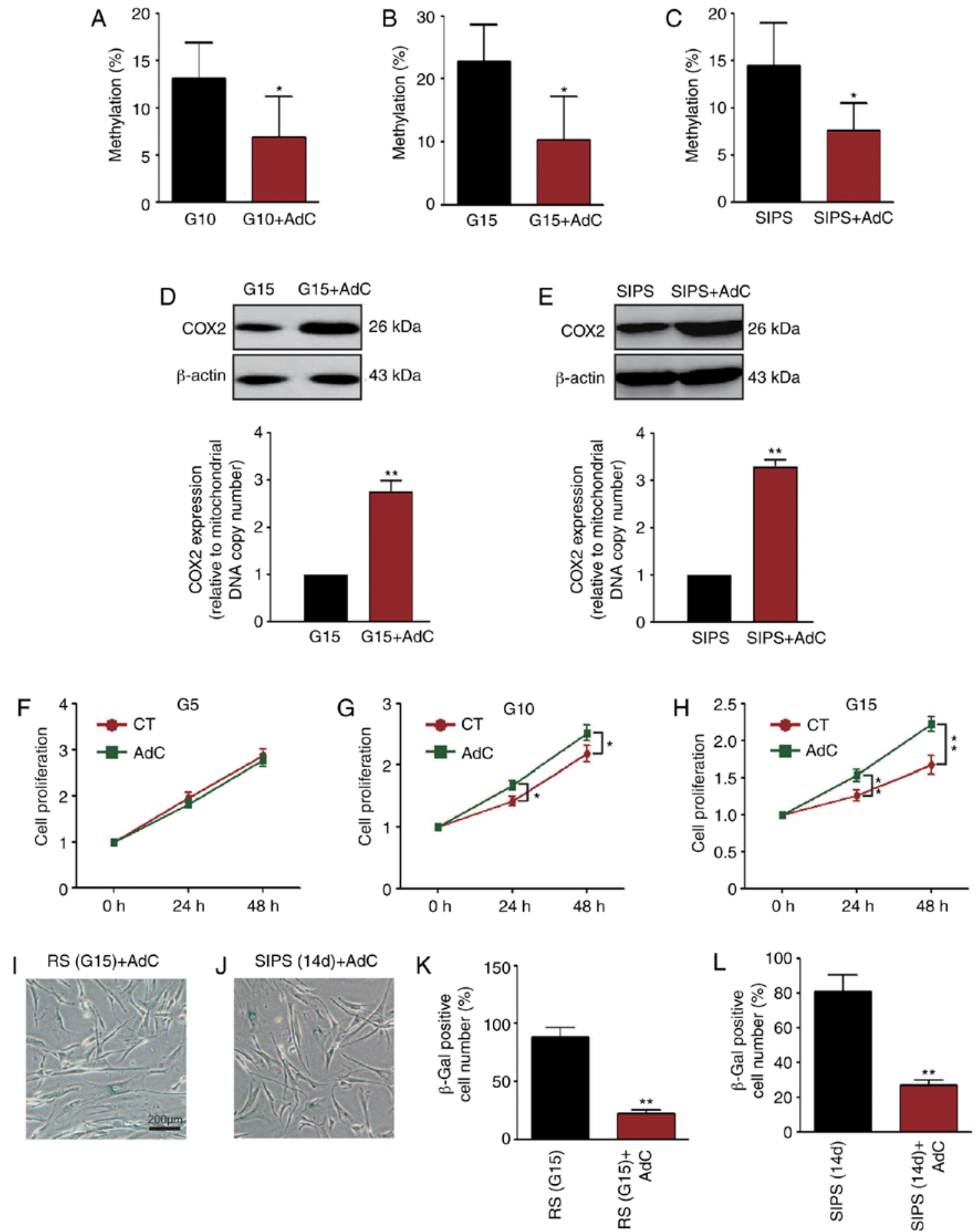

Figure 7. AdC decreases $C O X 2$ methylation and attenuates down HMSCs senescence. (A-C) COX2 methylation was decreased after HMSCs were treated with $1 \mu \mathrm{M}$ AdC. "P $<0.05$ as compared with the G10, G15 or SIPS group. (D and E) COX2 protein quantities were detected by western blot analysis and the expression levels were calculated. Statistical analysis results showing that the COX2 expression level increased after HMSCs were treated by AdC in both RS and SIPS cells. ${ }^{* *} \mathrm{P}<0.01$ as compared with the G15 or SIPS group. (F-H) WST-1 assay showing that AdC treatment led to the more rapid growth of G5, G10 and G15 HMSCs than those untreated at the same generation. The analysis time points were 24 and $48 \mathrm{~h} .{ }^{*} \mathrm{P}<0.05$ and ${ }^{* *} \mathrm{P}<0.01$ as compared with the CT groups. (I-L) SA- $\beta$-Gal staining results showing that AdC attenuates HMSC senescence. (I) HMSCs in the RS model; (J) HMSCs in the SIPS model; ( $\mathrm{K}$ and L) statistical analysis results of HMSCs stained positive for SA- $\beta-G a l .{ }^{* *} \mathrm{P}<0.01$ as compared with the RS (G15) and SIPS (14 days) groups shown in Fig. 1C and F. HMSCs, heart mesenchymal stem cells; RS, replicative senescence; SIPS, stress-induced premature senescence; AdC, 5-aza-2'-deoxycytidine.

compared and it was found that the trends of $p 16, p 27$ and $p 21$ were consistent between these two models, whereas that of $p 53$ was not. Additionally, telomere length shortening only occurred in RS and not in SIPS. These data suggest that although RS and SIPS share hallmarks of cellular senescence, such as $\beta$-Gal staining positivity, they still differ in some cytological features.
Although the exact mechanisms responsible for senescence are not currently fully understood, there is increasing evidence to suggest that the mitochondria act as central regulators of the aging process (27). In cardiac cells, which are the most energy-consuming cells in the human body, approximately one third of the cell volume is occupied 
by mitochondria (28). Similar to the methylation taking place in CpG sequences of nuclear DNA, methylation has also been discovered in mtDNA (29). Despite recent progress in the field of mtDNA methylation and its possible contribution to age-related diseases, clear-cut evidence of its functionality is still lacking (30).

In the present study, to reveal the mtDNA methylation status during the cell senescence process, a mitochondrial redox reaction-related gene, $\mathrm{COX} 2$, was selected, and its methylation levels were measured in both the RS and SIPS models. The results indicated that in the young G0 HMSCs, COX2 mtDNA exhibited a very low $\mathrm{CpG}$ methylation rate of $2.76 \%$. Following either continuous passaging or $\mathrm{H}_{2} \mathrm{O}_{2}$ exposure, the COX2 methylation levels in senescent HMSCs were significantly increased to 22.76 and $14.48 \%$. In young G0 HMSCs, there were 4 methylated $\mathrm{CpG}$ sites (4/29, Fig. 4), whereas in the G15 and SIPS HMSCs, the number of sites increased to 11 (11/29, Fig. 4) and 6 (6/29, Fig. 4). These data clearly indicate that $\mathrm{COX} 2$ methylation is a feature of the senescence of HMSCs. A previous study provided evidence that mtDNA methylation occurs not just at $\mathrm{CpG}$ sites: Dinucleotides $\mathrm{CpT}$, $\mathrm{CpA}$ and $\mathrm{CpC}$ were also methylated (31). Due to the limitations of technology and equipment, these data were not in the present study. However, the authors are still interested in investigating this phenomenon in future studies.

Mitochondrial gene expression is closely associated with mitochondria functionality. In the present study, it was demonstrated that the expression level of COX2 decreased following the senescence of HMSCs, and the addition of extraneous COX2 apparently delayed the aging process and promoted cell proliferation. These data suggest that the COX2 level is essential for maintaining HMSCs young. Normally, the D-loop is critical for the transcription and replication of mtDNA; thus, D-loop methylation is logically considered to control the mitochondrial gene expression (32). However, Ghosh et al demonstrated that methylcytosines distribute constantly across the mitochondria and that the methylcytosines near gene start sites can be temporally regulated, suggesting a potential regulatory role for the mitochondrial genome (33). Herein, it was demonstrated that there were 4 methylation hotspots on the $C O X 2$ gene during the aging process: The seventh $\mathrm{CpG}$, the 22nd CpG, the 27th $\mathrm{CpG}$ and the 29th CpG. However, to reveal whether these $\mathrm{CpGs}$ methylation are associated with $C O X 2$ downregulation and how they regulate $C O X 2$ expression, further investigations are warranted.

To further reveal the association between mtDNA methylation and cell senescence, AdC was used to treat the HMSCs. AdC is an inhibitor of Dnmt1, which has a mitochondrial targeting sequence and is the major enzyme responsible for the maintenance of DNA methylation (34). It was demonstrated that the administration of AdC may decrease the methylation and expression of COX2. Moreover, AdC also promoted proliferation and delayed the senescence of HMSCs. Mishra and Kowluru demonstrated that Dnmt1 binds directly at the D-loop and other regions (e.g., cytochrome b) of mtDNA and affects the transcription of mitochondrial genes, including COX2 (34). The present study yielded similar results to the study by Mishra and Kowluru. However, based on the current data, it still cannot be determined if the decrease in the COX2 level is regulated by the methylation of the D-loop or the methylation of itself, or both. Therefore, this unresolved issue will be the focus of future studies.

\section{Acknowledgements}

The authors would like to thank Dr Ji-Fan Hu and Professor Andrew R. Hoffman in Stanford University for providing technical advice on HMSC identification and culturing.

\section{Funding}

The present study was supported in part by grants from the Jilin International Collaboration Grant (no. 20180414065GH to DY and 20190701040GH to ZL), the Subject Arrangement Program from Science and Technology Department of Jilin Province (no. 20200201123JC to DY), the Youth Development Programme of Health Commission of Jilin Province (no. 2019Q007 to DY), the Health Special Fund from Jilin Province Department of Finance (no. 2018SCZWSZX-048 to XS), the National Natural Science Foundation of China (no. 81701270 to YL), the Transformation Fund of the First Hospital of Jilin University (no. JDYYZH-1902033 to YL), the China Postdoctoral Science Foundation (no. 171251 to LR), the Science-technology Foundation of the Jilin Provincial Education Department (no. JJKH20170823KJ to LR) and the Norman Bethune Program of Jilin University (no. 2015326 to LR).

\section{Availability of data and materials}

The datasets used and analyzed during the current study are available from the corresponding author on reasonable request.

\section{Authors' contributions}

DY contributed to the conception and design of the study. $\mathrm{XS}, \mathrm{ZW}, \mathrm{XC}$ and YL contributed to the acquisition, analysis and interpretation of the data. DY drafted the article. ZL and LR analyzed the data. TY was involved in performing the experiments. All authors have read and approved the final manuscript.

\section{Ethics approval and consent to participate}

Not applicable.

\section{Patient consent for publication}

Not applicable.

\section{Competing interests}

The authors declare that they have no competing interests.

\section{References}

1. Lakatta EG and Levy D: Arterial and cardiac aging: Major shareholders in cardiovascular disease enterprises: Part II: The aging heart in health: Links to heart disease. Circulation 107: 346-354, 2003. 
2. Soro-Arnaiz I, Li QOY, Torres-Capelli M, Meléndez-Rodríguez F, Veiga S, Veys K, Sebastian D, Elorza A, Tello D, HernansanzAgustín P, et al: Role of mitochondrial complex IV in age-dependent obesity. Cell Rep 16: 2991-3002, 2016.

3. Boengler K, Kosiol M, Mayr M, Schulz R and Rohrbach S: Mitochondria and ageing: Role in heart, skeletal muscle and adipose tissue. J Cachexia Sarcopenia Muscle 8: 349-369, 2017.

4. Frezza C: The role of mitochondria in the oncogenic signal transduction. Int J Biochem Cell Biol 48: 11-17, 2014.

5. Corsetti G, Pasini E, D'Antona G, Nisoli E, Flati V, Assanelli D, Dioguardi FS and Bianchi R: Morphometric changes induced by amino acid supplementation in skeletal and cardiac muscles of old mice. Am J Cardiol 101: E26-E34, 2008.

6. Cheng Z, Ito S, Nishio N, Thanasegaran S, Fang $\mathrm{H}$ and Isobe $\mathrm{K}$ : Characteristics of cardiac aging in C57BL/6 mice. Exp Gerontol 48: 341-348, 2013

7. Mozet C, Martin R, Welt K and Fitzl G: Cardioprotective effect of EGb 761 on myocardial ultrastructure of young and old rat heart and antioxidant status during acute hypoxia. Aging Clin Exp Res 21: 14-21, 2009.

8. El'darov ChM, Vays VB, Vangeli IM, Kolosova NG and Bakeeva LE: Morphometric examination of mitochondrial ultrastructure in aging cardiomyocytes. Biochemistry (Mosc) 80 : 604-609, 2015

9. Müller-Höcker J, Schäfer S, Weis S, Münscher C and Strowitzki T: Morphological-cytochemical and molecular genetic analyses of mitochondria in isolated human oocytes in the reproductive age. Mol Hum Reprod 2: 951-958, 1996.

10. Xin MG, Zhang J, Block ER and Patel JM: Senescence-enhanced oxidative stress is associated with deficiency of mitochondrial cytochrome c oxidase in vascular endothelial cells. Mech Ageing Dev 124: 911-919, 2003.

11. Abramson J, Svensson-Ek M, Byrne B and Iwata S: Structure of cytochrome c oxidase: A comparison of the bacterial and mitochondrial enzymes. Biochim Biophys Acta 1544: 1-9, 2001.

12. Yoshikawa S, Shinzawa-Itoh $\mathrm{K}$ and Tsukihara T: X-ray structure and the reaction mechanism of bovine heart cytochrome $\mathrm{c}$ oxidase. J Inorg Biochem 82: 1-7, 2000.

13. Stoccoro A, Siciliano G, Migliore L and Coppede F: Decreased methylation of the mitochondrial D-loop region in late-onse Alzheimer's disease. J Alzheimers Dis 59: 559-564, 2017.

14. Zheng LD, Linarelli LE, Brooke J, Smith C, Wall SS, Greenawald MH, Seidel RW, Estabrooks PA, Almeida FA and Cheng Z: Mitochondrial epigenetic changes link to increased diabetes risk and early-stage prediabetes indicator. Oxid Med Cell Longev 2016: 5290638, 2016.

15. Yu D, Du Z, Pian L, Li T, Wen X, Li W, Kim SJ, Xiao J, Cohen P, Cui J, et al: Mitochondrial DNA hypomethylation is a biomarker associated with induced senescence in human fetal heart mesenchymal stem cells. Stem Cells Int 2017: 1764549, 2017.

16. Manev $\mathrm{H}$ and $\mathrm{Uz} \mathrm{T}$ : DNA hypomethylating agents 5-aza-2'-deoxycytidine and valproate increase neuronal 5-lipoxygenase mRNA. Eur J Pharmacol 445: 149-150, 2002.

17. Saferali A, Lee J, Sin DD, Rouhani FN, Brantly ML and Sandford AJ: Longer telomere length in COPD patients with $\alpha 1$-antitrypsin deficiency independent of lung function. PLoS One 9: e95600, 2014.

18. el Bouazzaoui F, Henneman P, Thijssen P, Visser A, Koning F Lips MA, Janssen I, Pijl H, Willems van Dijk $\mathrm{K}$ and van Harmelen V: Adipocyte telomere length associates negatively with adipocyte size, whereas adipose tissue telomere length associates negatively with the extent of fibrosis in severely obese women. Int J Obes (Lond) 38: 746-749, 2014.
19. Livak KJ and Schmittgen TD: Analysis of relative gene expression data using real-time quantitative PCR and the 2(-Delta Delta C(T)) method. Methods 25: 402-408, 2001.

20. Ding Y, Xia BH, Zhang CJ and Zhuo GC: Mutations in mitochondrial tRNA genes may be related to insulin resistance in women with polycystic ovary syndrome. Am J Transl Res 9: 2984-2996, 2017

21. Rizvi S, Raza ST and Mahdi F: Telomere length variations in aging and age-related diseases. Curr Aging Sci 7: 161-167, 2014.

22. Lakatta EG and Sollott SJ: Perspectives on mammalian cardiovascular aging: Humans to molecules. Comp Biochem Physiol A Mol Integr Physiol 132: 699-721, 2002.

23. Hashimoto H, Olson EN and Bassel-Duby R: Therapeutic approaches for cardiac regeneration and repair. Nat Rev Cardiol 15: 585-600, 2018.

24. Hayflick L and Moorhead PS: The serial cultivation of human diploid cell strains. Exp Cell Res 25: 585-621, 1961.

25. Aan GJ, Hairi HA, Makpol S, Rahman MA and Karsani SA: Differences in protein changes between stress-induced premature senescence and replicative senescence states. Electrophoresis 34: 2209-2217, 2013

26. Kural KC, Tandon N, Skoblov M, Kel-Margoulis OV and Baranova AV: Pathways of aging: Comparative analysis of gene signatures in replicative senescence and stress induced premature senescence. BMC Genomics 17 (Suppl 14): S1030, 2016.

27. Payne BA and Chinnery PF: Mitochondrial dysfunction in aging: Much progress but many unresolved questions. Biochim Biophys Acta 1847: 1347-1353, 2015.

28. Bravo-Sagua R, Parra V, López-Crisosto C, Díaz P, Quest AF and Lavandero S: Calcium transport and signaling in mitochondria. Compr Physiol 7: 623-634, 2017.

29. Vanyushin BF and Kirnos MD: Structure of animal mitochondrial DNA (base composition, pyrimidine clusters, character of methylation). Biochim Biophys Acta 475: 323-336, 1977.

30. Zinovkina LA and Zinovkin RA: DNA methylation, mitochondria, and programmed aging. Biochemistry (Mosc) 80: 1571-1577, 2015.

31. Bellizzi D, D'Aquila P, Scafone T, Giordano M, Riso V, Riccio A and Passarino G: The control region of mitochondrial DNA shows an unusual $\mathrm{CpG}$ and non-CpG methylation pattern. DNA Res 20: 537-547, 2013

32. van der Wijst MG, van Tilburg AY, Ruiters MH and Rots MG: Experimental mitochondria-targeted DNA methylation identifies $\mathrm{GpC}$ methylation, not $\mathrm{CpG}$ methylation, as potential regulator of mitochondrial gene expression. Sci Rep 7: 177, 2017.

33. Ghosh S, Sengupta S and Scaria V: Comparative analysis of human mitochondrial methylomes shows distinct patterns of epigenetic regulation in mitochondria. Mitochondrion 18: 58-62, 2014.

34. Mishra M and Kowluru RA: Epigenetic modification of mitochondrial DNA in the development of diabetic retinopathy. Invest Ophthalmol Vis Sci 56: 5133-5142, 2015.

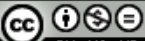

This work is licensed under a Creative Commons Attribution-NonCommercial-NoDerivatives 4.0 International (CC BY-NC-ND 4.0) License. 